\title{
LOCAL AND GLOBAL FACTORIZATIONS OF MATRIX-VALUED FUNCTIONS
}

BY

\author{
K. F. CLANCEY AND I. GOHBERG( $\left(^{1}\right)$
}

\begin{abstract}
Let $C$ be a simple closed Liapounov contour in the complex plane and $A$ an invertible $n \times n$ matrix-valued function on $C$ with bounded measurable entries. There is a well-known concept of factorization of the matrix function $A$ relative to the Lebesgue space $L_{p}(C)$. The notion of local factorization of $A$ relative to $L_{p}$ at a point $t_{0}$ in $C$ is introduced. It is shown that $A$ admits a factorization relative to $L_{p}(C)$ if and only if $A$ admits a local factorization relative to $L_{p}$ at each point $t_{0}$ in $C$. Several problems connected with local factorizations relative to $L_{p}$ are raised.
\end{abstract}

1. Let $C$ be a simple closed Liapounov contour in the complex plane $\mathbf{C}$ bounding the domain $D^{+}$. For our purposes it will entail no loss of generality to assume the domain $D^{+}$is bounded and that the complex number zero is in $D^{+}$. The notation $D^{-}$will be used for the domain complement to $D^{+} \cup C$ in $\mathrm{C} \cup\{\infty\}$.

An invertible continuous $n \times n$ matrix-valued function $A(t)(t \in C)$ is said to admit a (right standard) factorization relative to the contour $C$ in case $A=A_{-} U A_{+}$, where $A_{ \pm}$are invertible $n \times n$ matrix-valued functions that are analytic on $D^{ \pm}$, continuous on $D^{ \pm} \cup C$ and $U(t)=\operatorname{diag}\left[t^{\kappa_{1}}, \ldots, t^{\kappa_{n}}\right]$, where $\kappa_{1} \geqslant \cdots \geqslant \kappa_{n}$ are integers called the partial indices of $A$. Such a factorization of $A$ is not unique; however, the partial indices $\kappa_{1}, \ldots, \kappa_{n}$ are uniquely determined by $A$. An analogous definition of left standard factorization is possible. Here we will deal only with right factorization of matrixvalued functions.

A factorization of the above form does not exist for every invertible continuous matrix-valued function $A$, however, there are wide classes of matrix functions which admit such factorizations. For example, if the entries in $A$ satisfy a Lipschitz condition of order $\alpha(0<\alpha<1)$ on $C$, then $A$ admits a factorization relative to the contour $C$. Moreover, the factors $A_{ \pm}$have entries that satisfy a Lipschitz condition of the same order on $C$ [12]. If $C$ is the unit circle and the entries in $A$ have absolutely convergent Fourier series,

Received by the editors January 12, 1976.

AMS (MOS) subject classifications (1970). Primary 47B35; Secondary 45E05.

Key words and phrases. Operator factorizations, systems of singular integral equations.

$\left({ }^{1}\right)$ The work of both authors was supported in part by grants from the National Science Foundation. 
then the existence of a factorization, with the entries of $A_{ \pm}$possessing absolutely convergent Fourier series, is known [9]. Further developments in the theory of factorization of continuous matrix-valued functions appear in [7], [8], [1], and [2]. The concept of factorization has many applications. For example, such a factorization allows one to solve effectively systems of singular integral equations with continuous coefficients [8], [9] and [12].

Let $A(t)(t \in C)$ be an invertible continuous matrix-valued function on $C$. The function $A$ is said to possess a local factorization at the point $t_{0} \in C$ in case there is a neighborhood $N$ of $t_{0}$ in $\mathrm{C}$ and continuous matrix-valued functions $A_{ \pm}$on $\gamma=N \cap C$ such that $A=A_{-} A_{+}$on $\gamma$, where $A_{ \pm}$admit holomorphic continuations to $N^{ \pm}=D^{ \pm} \cap N$ with $\operatorname{det} A_{ \pm}(z) \neq 0, z \in N^{ \pm}$.

In [13] and [16] it was established that the continuous matrix-valued function $A$ admits a factorization relative to the contour $C$ if and only if $A$ admits a local factorization at each point $t_{0} \in C$. This allows one to factor even larger classes of continuous functions than those previously mentioned.

There are more general concepts of factorization of matrix-valued functions where the entries are assumed to be only bounded measurable functions on the contour $C$ (here measure will always refer to arc length measure). These notions of factorization are relative to spaces of functions on $C$. This paper is a study of the connections between local factorizations of these more general type and the respective global factorizations.

2. We now develop the definitions and notations that are necessary to discuss more general factorizations. Let $\gamma$ be a rectifiable arc in the complex plane. The notation $L_{p}^{n}(\gamma)\left[L_{p}^{n, n}(\gamma)\right](1 \leqslant p \leqslant \infty)$ will denote the usual Lebesgue spaces (with respect to arc length measure on $\gamma$ ) of $\mathbf{C}^{n}$-valued [ $n \times n$ matrix-valued] functions on $\gamma$. When the arc $\gamma$ is of Liapounov type, we define the customary singular integral operator on $L_{1}^{n}(\gamma)$ by

$$
(S f)(x)=\frac{1}{\pi i} \int_{\gamma} \frac{f(t)}{t-x} d t .
$$

It is well known that the operator $S$ defines a bounded linear operator on $L_{p}^{n}(\gamma)(1<p<\infty)$. The notation $W L_{p}^{n}(\gamma)$ will be employed for the class of measurable $n \times n$ matrix-valued functions $W$ such that the operator $f \rightarrow$ $W S W^{-1} f$ defines a bounded operator on $L_{p}^{n}(\gamma)(1<p<\infty)$.

Let $\Omega$ be a bounded simply connected open set in $C$. It will always be assumed that the boundary $\Gamma$ of $\Omega$ is a piecewise Liapounov contour. The class $E_{p}(\Omega)(1<p<\infty)$ consists of those analytic functions $f$ on $\Omega$ that satisfy $\sup _{k}\left(\int_{\Gamma_{k}}|f(z)|^{p}|d z|\right)<\infty$, where $\left\{\Gamma_{k}\right\}_{k=1}^{\infty}$ is a system of closed rectifiable contours that eventually surround every compact subset of $\Omega$. The notation $E_{\infty}(\Omega)$ will be used for the space of bounded analytic functions on $\Omega$.

In the next section a discussion is given of the properties of the spaces 
$E_{p}(\Omega)(1 \leqslant p<\infty)$. Here we remark only that an element $f \in E_{p}(\Omega)(1 \leqslant p$ $\leqslant \infty$ ) has nontangential limiting values at almost every point of $\Gamma$ and this nontangential limit function (still denoted by $f$ ) is in $L_{p}(\Gamma)$. The space $\left[L_{p}(\Gamma)\right]^{+}$is the (closed) subspace of $L_{p}(\Gamma)$ consisting of functions which are nontangential limit functions of elements in $E_{p}(\Omega)(1 \leqslant p \leqslant \infty)$. A similar definition is made for the spaces $\left[L_{p}(\Gamma)\right]^{-}$by considering the analogous spaces of analytic functions on $\Omega^{\prime}=[C-(\Omega \cup \Gamma)] \cup\{\infty\}$. We will denote by $E_{p}^{n, n}(\Omega)\left(\left[L_{p}^{n, n}(\Gamma)\right]^{ \pm}\right)$the space of $n \times n$ matrices with entries from $E_{p}(\Omega)\left(\left[L_{p}(\Gamma)\right]^{ \pm}\right)$.

We remark that if $C$ is the Liapounov contour introduced earlier, then the space $\left[L_{p}^{n, n}(C)\right]^{+}$coincides with the range of the projector $P=\frac{1}{2}[I+S]$ on $L_{p}^{n, n}(C)$, and $\left[L_{p}^{n, n}(C)\right]^{-}$is the span of the range of the projector $Q=\frac{1}{2}[I-$ $S$ ] and the constant matrices in $L_{p}^{n, n}(C)(1<p<\infty)$.

Let $A$ be an invertible element in $L_{\infty}^{n, n}(C), p$ a fixed real number $(1<p<$ $\infty)$ and $q$ the conjugate exponent $\left(p^{-1}+q^{-1}=1\right)$. The matrix function $A$ is said to admit a factorization relative to $L_{p}(C)$ in case:

(i) $A=A_{-} U A_{+}$, where $A_{+} \in\left[L_{q}^{n, n}(C)\right]^{+}, A_{-} \in\left[L_{p}^{n, n}(C)\right]^{-}, A_{+}^{-1} \in$ $\left[L_{p}^{n, n}(C)\right]^{+}, A_{-}^{-1} \in\left[L_{q}^{n, n}(C)\right]^{-}$and $U(t)=\operatorname{diag}\left(t^{\kappa_{1}}, \ldots, t^{\kappa_{n}}\right)$, where $\kappa_{1}$ $>\cdots>\kappa_{n}$ are integers called the partial indices of $A$.

(ii) The function $A_{-}$is in $W L_{p}^{n}(C)$.

Factorizations relative to $L_{p}(C)$ play a decisive role in the study of the Fredholm behavior of singular integral operators on $L_{p}^{n}(C)$ of the form

$$
(T f)(x)=E(x) f(x)+\frac{F(x)}{\pi i} \int_{C} \frac{f(t)}{t-x} d t,
$$

here $E$ and $F$ are elements in $L_{\infty}^{n, n}(C)$ (see, e.g., [10], [15] and [17]). For example, if $A=(E-F)^{-1}(E+F)$ admits a factorization relative to $L_{p}(C)$ of the form $A=A_{-} A_{+}$(so that the partial indices of $A$ are all zero), then the operator $T$ is invertible with $T^{-1}$ given by

$$
T^{-1}=(E+F)^{-1}\left[E+F A_{-} S A_{-}^{-1}\right][E-F]^{-1} \text {. }
$$

The form of the operator $T^{-1}$ can serve as an explanation of condition (ii) in the definition of factorization.

3. Let $\gamma$ be an arc on $C$ and $p$ a fixed real number $(1<p<\infty)$. We will say the $n \times n$ matrix-valued function $A_{ \pm}$is in $\left[L_{p}^{n, n}(\gamma)\right]^{ \pm}$in case $\gamma$ is part of the boundary of a simply connected domain $\Omega^{ \pm} \subset D^{ \pm}$(with the boundary of $\Omega^{ \pm}$being a piecewise Liapounov contour) and $A_{ \pm}$is the restriction to $\gamma$ of the nontangential limit function of an element in $E_{p}^{n, n}\left(\Omega^{ \pm}\right)$.

Let $A$ be an invertible element in $L_{\infty}^{n, n}(C), p$ a fixed real number $(1<p<$ $\infty), q$ the conjugate exponent and $t_{0} \in C$. The matrix function $A$ is said to admit a local factorization relative to $L_{p}$ at $t_{0}$ in case the point $t_{0}$ is in the interior of a subarc $\gamma_{0} \subset C$ and such that: 
(I) $A={ }^{0} A_{-}{ }^{0} A_{+}$on $\gamma_{0}$, where ${ }^{0} A_{+} \in\left[L_{q}^{n, n}\left(\gamma_{0}\right)\right]^{+},{ }^{0} A_{-} \in\left[L_{p}^{n, n}\left(\gamma_{0}\right)\right]^{-}$, ${ }^{0} A_{+}^{-1} \in\left[L_{p}^{n, n}\left(\gamma_{0}\right)\right]^{+}$and ${ }^{0} A_{-}^{-1} \in\left[L_{q}^{n, n}\left(\gamma_{0}\right)\right]^{-}$.

(II) The function ${ }^{0} A$ _is in $W L_{p}\left(\gamma_{0}\right)$.

It is natural to expect that the local factorization defined above would have the same properties on subarcs of $\gamma_{0}$. We will see that this is the case. The proof of this fact will involve the theory of Carleson measures on the unit disc.

The main result of this paper is the following:

THEOREM. Let $A$ be an invertible element in $L_{\infty}^{n, n}(C)$ and $p$ fixed $(1<p<$ $\infty)$. The function $A$ admits a factorization relative to $L_{p}(C)$ if and only if the function $A$ admits a local factorization relative to $L_{p}$ at each point $t_{0} \in C$.

The proof of the above theorem is presented in \$2. \$1 deals with preliminaries. The final section (\$3) contains a discussion of the result in this theorem along with other unsolved local and global problems.

\section{Preliminaries.}

1. Let $\Omega$ be a bounded simply connected open subset of the plane with boundary $\Gamma$ a piecewise Liapounov contour which has positive orientation with respect to $\Omega$. In this section we will discuss some properties of the spaces $E_{p}(\Omega)(1<p<\infty)$. These properties will be used in the next section to prove the theorem stated in the preceding section. Most of these properties are discussed in [6] and [11]. We emphasize that the restriction which has been placed on the boundary of $\Omega$ implies that $\Omega$ is a Smirnov domain (see, e.g., [11, p. 444]). Some of the properties stated below do not hold in case the domain $\boldsymbol{\Omega}$ is not Smirnov. The notation $\varphi$ will be used for a fixed conformal mapping of the unit disc $\mathbf{D}$ onto $\Omega$ and $\psi$ will denote the inverse of this mapping. In case $\Omega=\mathrm{D}$, we will use the more customary notation $H^{p}$ for the space $E_{p}(\mathrm{D})$ $(1<p<\infty)$.

The following four properties of the spaces $E_{p}(\Omega)$ are well known:

$1^{\circ}$. A function $f$ analytic on $\Omega$ belongs to $E_{p}(\Omega)$ if and only if the function $F(\omega)=f(\varphi(\omega))\left(\varphi^{\prime}(\omega)\right)^{1 / p}(|\omega|<1)$ belongs to $H^{p}(1 \leqslant p \leqslant \infty)$. Indeed every function $F$ in $H^{p}$ has the form $F=f \circ \varphi\left[\varphi^{\prime}\right]^{1 / p}$ for some $f \in E_{p}(\Omega)(1<p<$ $\infty)$.

$2^{\circ}$. Let $f \in E_{p}(\Omega)(1<p<\infty)$. The function $f$ has a nontangential limiting value at almost every point of $\Gamma$. Further, the function $f$ defined on $\Gamma$ via these nontangential limiting values satisfies $f \in L_{p}(\Gamma)$. The function $f$ can be recovered at $z \in \Omega$ from its limiting values on $\Gamma$ by Cauchy's integral formula

$$
f(z)=\frac{1}{2 \pi i} \int_{\Gamma} \frac{f(t)}{t-z} d t .
$$

Moreover, this latter integral vanishes for $z \notin \Omega \cup \Gamma$. 
$3^{\circ}$. A function $g \in L_{p}(\Gamma)(1 \leqslant p \leqslant \infty)$ equals a.e. the nontangential limit function of an element in $E_{p}(\Omega)$ if and only if the integral $(2 \pi i)^{-1} \int_{\Gamma} g(t)(t-$ $z)^{-1} d t$ vanishes for $z \notin \Omega \cup \Gamma$. In the case where this latter integral vanishes for $z \notin \Omega \cup \Gamma$, then it defines on $\Omega$ a function $g \in E_{p}(\Omega)$ whose nontangential limiting values agree with $g$ a.e. on $\Gamma$.

$4^{\circ}$. The collection $\left[L_{p}(\Gamma)\right]^{+}(1 \leqslant p \leqslant \infty)$ consisting of functions in $L_{p}(\Gamma)$ which are the nontangential limit functions of elements in $E_{p}(\Omega)$ is a closed subspace of $L_{p}(\Gamma)$. In case $1 \leqslant p<\infty,\left[L_{p}(\Gamma)\right]^{+}$is the closure in $L_{p}(\Gamma)$ of the matrix functions with polynomial entries.

2. It is known that if $\Gamma$ (the boundary of $\Omega$ ) is a Liapounov contour, then the conformal mapping $\psi$ is smooth on $\Omega \cup \Gamma$ (see [11, p. 426]). More generally we observe:

Let $\gamma$ be a Liapounov subarc of $\Gamma$. The conformal mapping $\psi$ is smooth on $\Omega \cup \Gamma$ in a neighborhood of every point on $\gamma$.

To see this last remark we proceed as follows. Let $t_{0}$ be in $\gamma$. Choose a subdomain $\Omega_{0}$ in $\Omega$ whose boundary is a Liapounov contour $\Gamma_{0}$ such that $t_{0}$ is in the interior of $\Gamma \cap \Gamma_{0}$. Let $\alpha$ be a conformal mapping of $\psi\left(\Omega_{0}\right)$ onto $\mathbf{D}$. The composition $\alpha \circ \psi$ is a conformal map of $\Omega_{0}$ to $\mathbf{D}$ and therefore, $\alpha \circ \psi$ is smooth on $\Omega_{0} \cup \Gamma_{0}$. Since $\alpha$ is conformal on a neighborhood of an arc of the unit circle containing $\psi\left(t_{0}\right)$, then $\psi$ must be smooth on $\Omega \cup \Gamma$ in a neighborhood of $t_{0}$.

The following question will arise naturally in the ensuing arguments. Let $\Omega^{\prime} \subset \Omega$ be a simply connected open set with piecewise Liapounov boundary $\Gamma^{\prime}$. Let $f \in E_{p}(\Omega)(1 \leqslant p \leqslant \infty)$. When is the restriction of $f$ to $\Omega^{\prime}$ in $E_{p}\left(\Omega^{\prime}\right)$ ?

The answer is clear when $p=\infty$ or in the case where $\Omega^{\prime} \cup \Gamma^{\prime} \subset \Omega$. A subdomain $\Omega^{\prime} \subset \Omega$ which has the property that whenever $f$ belongs to $E_{p}(\Omega)$ $(1 \leqslant p \leqslant \infty)$, then the restriction of $f$ to $\Omega^{\prime}$ is in $E_{p}\left(\Omega^{\prime}\right)$ will be called a normal subdomain of $\Omega$.

We will be concerned here only with the case where $\Gamma \cap \Gamma^{\prime}$ is an $\operatorname{arc} \gamma$. The notation $\gamma=\left[t_{1}, t_{2}\right]$ will be used to indicate the endpoints $t_{1}, t_{2}$ of the arc $\gamma$. It will always be assumed that the points $t_{1}$ and $t_{2}$ are in the interior of Liapounov subarcs of $\Gamma$. As we have observed, this latter condition ensures that the conformal mapping $\psi$ is smooth on $\Omega \cup \Gamma$ in neighborhoods of $t_{1}$ and $t_{2}$. The arc $\Gamma^{\prime} \backslash \gamma$ will be denoted by $\gamma^{\prime}$.

It follows from property $4^{\circ}$ that if there is a constant $K>0$ such that

$$
\int_{\gamma^{\prime}}|f(z)|^{p}|d z| \leqslant K \int_{\Gamma}|f(z)|^{p}|d z|
$$

for all $f \in E_{p}(\Omega)(1 \leqslant p \leqslant \infty)$, then $\Omega^{\prime}$ is a normal subdomain of $\Omega$.

In view of the Cauchy representation of functions in $E_{p}(\Omega)(1 \leqslant p \leqslant \infty)$, it is clear that the existence of a constant $K$ such that inequality (1) holds, depends only on the behavior of $\gamma^{\prime}$ near the points $t_{1}$ and $t_{2}$. In fact we can 
consider the following problem. Let $\gamma_{0}$ be a smooth arc parametrized by $\eta$ : $[0,1] \rightarrow \mathrm{C}$, where $\eta(t) \in \Omega(0 \leqslant t<1)$ and $\eta(1)=t_{0} \in \Gamma$. Assume $t_{0}$ is in the interior of a Liapounov subarc. Does there exist a constant $K>0$ such that

$$
\int_{\gamma_{0}}|f(z)|^{p}|d z| \leqslant K \int_{\Gamma}|f(z)|^{p}|d z|
$$

for all $f \in E_{p}(\Omega)(1 \leqslant p<\infty)$ ?

We will presently see that this last question has an affirmative answer. It follows from property $1^{\circ}$ that the answer is yes if and only if there is a constant $K>0$ such that

$$
\int_{\psi\left(\gamma_{0}\right)}|g(z)|^{p}|d z| \leqslant K \int_{0}^{2 \pi}\left|g\left(e^{i \theta}\right)\right|^{p} \frac{d \theta}{2 \pi}
$$

for all $g \in H^{p}(1 \leqslant p<\infty)$.

A result of Carleson (see, e.g., [6, p. 157]) gives a necessary and sufficient condition for the existence of a constant $K$ so that (3) holds. We now describe this result.

Let $\omega$ be a nonnegative measure on the unit disc D. Let $I=\left\{e^{i \theta}:\left|\theta-\theta_{0}\right|\right.$ $\leqslant \delta\}$ be a subarc of the unit circle and $R(I)=\left\{r e^{i \theta}: 1-\delta<r<1\right.$, $\left.e^{i \theta} \in I\right\}$. The measure $\mu$ is said to be a Carleson measure on $\mathbf{D}$ in case

$$
\sup _{|I|<2 \pi} \frac{\mu(R(I))}{|I|}<\infty,
$$

where $|I|$ denotes the length of $I$.

The theorem of Carleson establishes that there is a constant $K>0$ such that inequality (3) holds for all $g \in H^{p}(1 \leqslant p<\infty)$ if and only if arc length measure on $\psi\left(\gamma_{0}\right)$ is a Carleson measure. To see that arc length measure on $\psi\left(\gamma_{0}\right)$ is a Carleson measure it is only necessary to realize that the arc $\psi\left(\gamma_{0}\right)$ near $\psi\left(t_{0}\right)$ is either the graph of a smooth function over an arc of the unit circle containing $\psi\left(t_{0}\right)$ or the graph of a smooth function over the radius of the unit circle terminating at $\psi\left(t_{0}\right)$.

We summarize the above discussion with the following:

$5^{\circ}$. Let $\Omega^{\prime} \subset \Omega$ be a simply connected open set with piecewise Liapounov boundary $\Gamma^{\prime}$. Assume that $\Gamma \cap \Gamma^{\prime}$ is an arc $\gamma=\left[t_{1}, t_{2}\right]$, where $t_{1}$ and $t_{2}$ are points in the interior of Liapounov subarcs of $\Gamma$. Then $\Omega^{\prime}$ is a normal subdomain of $\Omega$.

\section{Proof of the theorem.}

1. Throughout this section $A$ will denote a fixed invertible element in $L_{\infty}^{n, n}(C), p$ will be a fixed real number satisfying $1<p<\infty$ and $q$ will denote the conjugate exponent. In this section we will show that if $A$ admits a local factorization relative to $L_{p}$ at each point $t_{0}$ in $C$, then $A$ admits a factorization relative to $L_{p}(C)$. 
It will be convenient to make the following definition. Let $\gamma$ be a subarc of $C$. The matrix function $A$ is said to admit a local factorization relative to $L_{p}$ on the arc $\gamma$ in case there are domains $\Omega^{ \pm} \subset D^{ \pm}$with piecewise Liapounov boundaries $\Gamma^{ \pm}$such that $\Gamma^{ \pm} \cap C=\gamma$ and $A=A_{-} A_{+}$on $\gamma$ where

(i) $A_{+} \in E_{q}^{n, n}\left(\Omega^{+}\right), A_{-} \in E_{p}^{n, n}\left(\Omega^{-}\right), A_{+}^{-1} \in E_{p}^{n, n}\left(\Omega^{+}\right)$and $A_{-}^{-1} \in$ $E_{q}^{n, n}\left(\Omega^{-}\right)$.

(ii) The function $A_{-}$is in $W L_{p}^{n}(\gamma)$.

As a preliminary step we indicate that if $A$ admits a local factorization relative to $L_{p}$ at a point $t_{0}$ in $C$, then $A$ admits a local factorization relative to $L_{p}$ on an arc $\gamma_{0}$ containing $t_{0}$ in its interior. Recall that if $A$ admits a local factorization at the point $t_{0} \in C$ relative to $L_{p}$, then there is an arc $\gamma_{0}$ on $C$ with $t_{0}$ an interior point of $\gamma_{0}$ such that $A={ }^{0} A_{-}{ }^{0} A_{+}$on $\gamma_{0}$ where ${ }^{0} A_{+} \in$ $\left[L_{q}^{n, n}\left(\gamma_{0}\right)\right]^{+},{ }^{0} A_{-} \in\left[L_{p}^{n, n}\left(\gamma_{0}\right)\right]^{-},{ }^{0} A_{+}^{-1} \in\left[L_{p}^{n, n}\left(\gamma_{0}\right)\right]^{+}$and ${ }^{0} A_{-}^{-1} \in\left[L_{q}^{n, n}\left(\gamma_{0}\right)\right]^{-}$. The statements ${ }^{0} A_{+} \in\left[L_{q}^{n, n}\left(\gamma_{0}\right)\right]^{+}$and ${ }^{0} A_{+}^{-1} \in\left[L_{p}^{n, n}\left(\gamma_{0}\right)\right]^{+}$mean that there are domains $\Omega_{1}^{+} \subset D^{+}, \Omega_{2}^{+} \subset D^{+}$with $\gamma_{0}$ a subset of the boundaries of $\Omega_{i}^{+}$ $(i=1,2)$ and functions $F \in E_{q}^{n, n}\left(\Omega_{1}^{+}\right), G \in E_{p}^{n, n}\left(\Omega_{2}^{+}\right)$such that $F={ }^{0} A_{+}$ and $G={ }^{0} A_{+}^{-1}$ on $\gamma_{0}$. A priori there is no reason to assume the domains $\Omega_{1}^{+}$ and $\Omega_{2}^{+}$are the same, however, one consequence of property $5^{\circ}$ is that by shrinking $\gamma_{0}$ (if necessary), then the equality $\Omega_{1}^{+}=\Omega_{2}^{+}$can be achieved. In fact one can choose this common domain $\Omega_{0}^{+}$so that its boundary in $D^{+}$is a polygonal curve. It follows from Privalov's uniqueness theorem (see, e.g., [11]) that the functions $F$ and $G$ are uniquely determined by the values on $\gamma_{0}$ of ${ }^{0} A_{+}$and ${ }^{0} A_{+}^{-1}$, respectively. Further the function $F G$ belongs to $E_{1}^{n, n}\left(\Omega_{0}^{+}\right)$and has nontangential limiting values equal to the identity matrix on $\gamma_{0}$. Again using Privalov's uniqueness theorem it follows that $G=F^{-1}$ on $\Omega_{0}^{+}$. Similar arguments and assumptions can be made concerning the relationship of the functions ${ }^{0} A_{-}$and ${ }^{0} A_{-}^{-1}$. The reasoning above shows that whenever $A$ admits a local factorization relative to $L_{p}$ at a point $t_{0} \in C$, then $A$ admits a local factorization relative to $L_{p}$ on an arc $\gamma_{0}$ containing $t_{0}$ as an interior point.

It should be observed that if there exist two local factorizations $A=$ $A_{-} A_{+}$and $A=B_{-} B_{+}$relative to $L_{p}$ on an arc $\gamma$, then the smoothness properties of the corresponding factors at interior points of the arc are the same. More precisely there exists an invertible holomorphic function $H$ on the interior of $\gamma$ such that $A_{-}=B_{-} H$ and $A_{+}=H^{-1} B_{+}$.

2. We need the following simple lemma on $W L_{p}^{n}(\gamma)$.

LEMMA 1. Let $\gamma$ be a Liapounov arc. Suppose that $W \in W L_{p}^{n}(\gamma)$, where $W \in L_{p}^{n, n}(\gamma), W^{-1} \in L_{q}^{n, n}(\gamma)$ and $H$ is an invertible $n \times n$ matrix-valued function on $\gamma$ with smooth entries. Then $W H$ is in $W L_{p}^{n}(\gamma)$.

Proof. We write $W H S(W H)^{-1}=W S W^{-1}+W S K W^{-1}$, where $K(x, t)$ $=H(x)\left[H^{-1}(t)-H^{-1}(x)\right]$. Since, $K(x, t)(t-x)^{-1}$ is bounded on $\gamma \times \gamma$, 
$W$ belongs to $L_{p}^{n, n}(\gamma)$ and $W^{-1} \in L_{q}^{n, n}(\gamma)$, then the operator $W S K W^{-1}$ is bounded on $L_{p}^{n}(\gamma)$. This completes the proof.

An immediate consequence of the preceding lemma is the following. Suppose $A=A_{-} U A_{+}$is a factorization of $A$ relative to $L_{p}(C)$. Then the operator $f \rightarrow A_{-} S A_{-}^{-1} f$ is bounded on $L_{p}^{n}(C)$ if and only if the operator $f \rightarrow A_{+}^{-1} S A_{+} f$ is bounded on $L_{p}^{n}(C)$. It is not hard to show that the condition $A_{-} \in W L_{p}^{n}(C)$ is also equivalent to the boundedness of the operator $f \rightarrow A_{+}^{-1} S A_{-}^{-1} f$ on $L_{p}^{n}(C)$.

It follows that in the definition of factorization relative to $L_{p}(C)$ the condition $A_{-} \in W L_{p}^{n}(C)$ can be replaced by either of the conditions $f \rightarrow$ $A_{+}^{-1} S A_{+} f$ is bounded on $L_{p}^{n}(C)$ or $f \rightarrow A_{+}^{-1} S A_{-}^{-1} f$ is bounded on $L_{p}^{n}(C)$. This last statement is true as well for local factorizations relative to $L_{p}$.

3. Next we prove the following.

LemMa 2. Let $\gamma_{1}$ and $\gamma_{2}$ be open subarcs of $C$ such that $\gamma_{1} \cap \gamma_{2} \neq \varnothing$. Assume that $A$ admits a local factorization relative to $L_{p}$ on both $\gamma_{1}$ and $\gamma_{2}$. Then $A$ admits a local factorization relative to $L_{p}$ on the union of $\gamma_{1}$ and $\gamma_{2}$.

Proof. We give the details of the proof only for the case where $\gamma_{1} \cup \gamma_{2}$ is not all of $C$. The argument for the case where $\gamma_{1} \cup \gamma_{2}=C$ is similar. The arc $\gamma_{i}$ is part of the boundary of simply connected domains $\Omega_{i}^{ \pm} \subset D^{ \pm}$, where the boundary of $\Omega_{i}^{ \pm}$are piecewise Liapounov contours $(i=1,2)$. On $\gamma_{i}$ we have that $A={ }^{i} A_{-}{ }^{i} A_{+}$where ${ }^{i} A_{+} \in E_{q}^{n, n}\left(\Omega_{i}^{+}\right),{ }^{i} A_{-} \in E_{p}^{n, n}\left(\Omega_{i}^{-}\right),{ }^{i} A_{+}^{-1} \in$ $E_{p}^{n, n}\left(\Omega_{i}^{+}\right),{ }^{i} A_{-}^{-1} \in E_{q}^{n, n}\left(\Omega_{i}^{-}\right)$and ${ }^{i} A_{-} \in W L_{p}^{n}\left(\gamma_{i}\right)(i=1,2)$.

On the arc $\gamma=\gamma_{1} \cap \gamma_{2}$ we have the identity ${ }^{1} A_{-}^{-1}{ }^{2} A_{-}={ }^{1} A_{+}{ }^{2} A_{+}^{-1}$. Consider the function

$$
H(z)= \begin{cases}{ }^{1} A_{+}{ }^{2} A_{+}^{-1}(z), & z \in \Omega_{1}^{+} \cap \Omega_{2}^{+}, \\ { }^{1} A_{-}^{-1}{ }^{2} A_{-}(z), & z \in \Omega_{1}^{-} \cap \Omega_{2}^{-} .\end{cases}
$$

The function $H$ is analytic across a subarc $\gamma^{\prime}$ of $\gamma$. To see this choose $\gamma^{\prime} \subset \gamma$ to be part of the boundary of domains $\Omega_{3}^{ \pm} \subset \Omega_{1}^{ \pm} \cap \Omega_{2}^{ \pm}$such that $\Omega_{3}^{ \pm}$is a normal subdomain of $\Omega_{1}^{ \pm}$and $\Omega_{2}^{ \pm}$. The restriction of $H$ to $\Omega_{3}^{+}$is in $E_{1}^{n, n}\left(\Omega_{3}^{+}\right)$ and the restriction of $H$ to $\Omega_{3}$ is in $E_{1}^{n, n}\left(\Omega_{3}^{-}\right)$. The fact that $H$ is analytic across $\gamma^{\prime}$ follows from the Cauchy integral representation of functions in $E_{1}^{n, n}\left(\Omega_{3}^{ \pm}\right)$. Clearly, we can assume that $H$ is an invertible holomorphic matrix-valued function in a neighborhood of $\gamma^{\prime}$.

Let $t_{0}$ be a fixed point in the interior of $\gamma^{\prime}$. Choose a simple closed contour $C^{\prime}$ that cuts $C$ perpendicularly at $t_{0}$ (see Figure 1). Let $H^{\prime}$ be an invertible Hölder continuous extension of the function $H$ to the contour $C^{\prime}$ which agrees with $H$ in a neighborhood of $t_{0}$ on $C^{\prime}$. We can choose $H^{\prime}$ so that $H^{\prime}$ admits a factorization $H^{\prime}=H_{-} H_{+}$relative to $C^{\prime}$. 


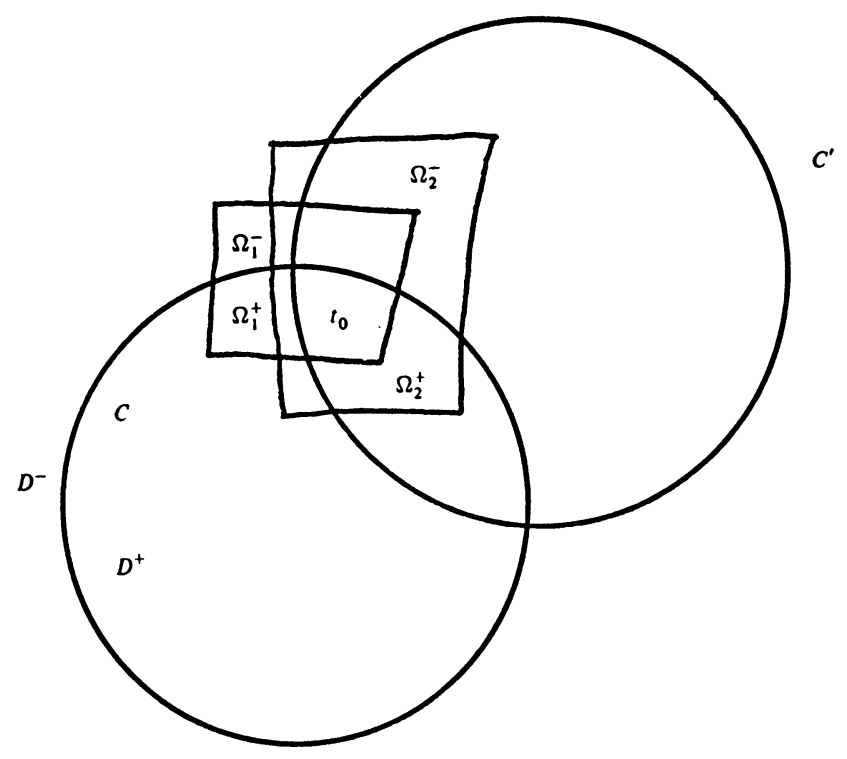

FIGURE 1

Define the functions

$$
{ }^{0} A_{+}(z)= \begin{cases}H_{-}^{-1}(z)^{1} A_{+}(z), & z \in \Omega_{1}^{+} \cap \operatorname{ext}\left(C^{\prime}\right), \\ H_{+}(z)^{2} A_{+}(z), & z \in \Omega_{2}^{+} \cap \operatorname{int}\left(C^{\prime}\right),\end{cases}
$$

and

$$
{ }^{0} A_{-}(z)= \begin{cases}{ }^{1} A_{-}(z) H_{-}(z), & z \in \Omega_{1}^{-} \cap \operatorname{ext}\left(C^{\prime}\right), \\ { }^{2} A_{-}(z) H_{+}^{-1}(z), & z \in \Omega_{2}^{-} \cap \operatorname{int}\left(C^{\prime}\right) .\end{cases}
$$

The function ${ }^{0} A_{+}$is analytic across the arc of $C^{\prime}$ in $D^{+}$where $H^{\prime}=H$. Similarly, the function ${ }^{0} A_{-}$is analytic across the arc of $C^{\prime}$ in $D^{-}$where $H^{\prime}=H$. Using property $5^{\circ}$ of the preceding section, we can choose simply connected domains $\Omega_{0}^{ \pm} \subset D^{ \pm}$where the boundaries of $\Omega_{0}^{ \pm}$are piecewise Liapounov contours containing $\gamma_{0}$ and such that $A={ }^{0} A_{-}{ }^{0} A_{+}$on $\gamma_{0}$ with ${ }^{0} A_{+} \in E_{q}^{n, n}\left(\Omega_{0}^{+}\right),{ }^{0} A_{-} \in E_{p}^{n, n}\left(\Omega_{0}^{-}\right),{ }^{0} A_{+}^{-1} \in E_{p}^{n, n}\left(\Omega_{0}^{+}\right)$and ${ }^{0} A_{-}^{-1} \in E_{q}^{n, n}\left(\Omega_{0}^{-}\right)$. To complete the proof of the lemma we need only establish that ${ }^{0} A_{-}$is in $W L_{p}^{n}\left(\gamma_{0}\right)$.

First we remark that the functions $H_{ \pm}$admit analytic continuations to a neighborhood $\gamma^{\prime \prime}$ of $t_{0}$ where $\gamma^{\prime \prime} \subset \gamma^{\prime}$. This is because of the fact that $H^{\prime}$ is analytic in a neighborhood of $t_{0}$. We can assume by shrinking to a subarc that $H_{ \pm}$are analytic in a neighborhood of $\gamma^{\prime \prime}$. We denote the arcs $\gamma_{1}, \gamma_{2}, \gamma^{\prime \prime}$ by $\gamma_{1}=\left(z_{0}, z_{1}\right), \gamma_{2}=\left(z_{0}^{\prime}, z_{1}^{\prime}\right)$ and $\gamma^{\prime \prime}=\left(z_{2}, z_{3}\right)$, respectively. If $E$ is a subset of $C$, 
then $M_{E}$ will denote the operator of multiplication by the characteristic function of $E$.

We note that

$$
{ }^{0} A_{-} S^{0} A_{-}^{-1}=M_{\left(z_{0}, t_{0}\right)}{ }^{0} A_{-} S^{0} A_{-}^{-1}+M_{\left(t_{0, z_{1}^{\prime}}\right)}{ }^{0} A_{-} S^{0} A_{-}^{-1} \text {. }
$$

By symmetry it suffices to show that the operator $M_{\left(z_{0}, t_{0}\right)}{ }^{0} A_{-} S^{0} A_{-}^{-1}$ is bounded on $L_{p}^{n}\left(\gamma_{0}\right)$. We write

$$
M_{\left(z_{0}, t_{0}\right)}{ }^{0} A_{-} S^{0} A_{-}^{-1}=M_{\left(z_{0}, t_{0}\right)}{ }^{0} A_{-} S^{0} A_{-}^{-1} M_{\left(z_{0, z_{3}}\right)}+M_{\left(z_{0,0}, t_{0}\right)}{ }^{0} A_{-} S^{0} A_{-}^{-1} M_{\left(z_{3}, z_{1}\right)} \text {. }
$$

It follows from the facts ${ }^{0} A_{-} \in L_{p}^{n, n}\left(\gamma_{0}\right)$ and ${ }^{0} A_{-}^{-1} \in L_{q}^{n, n}\left(\gamma_{0}\right)$ that the second operator on the right side of this last expression is bounded. Further,

$$
M_{\left(z_{0,}, t_{0}\right)}{ }^{0} A_{-} S^{0} A_{-}^{-1} M_{\left(z_{0}, z_{3}\right)}=M_{\left(z_{0}, t_{0}\right)} A_{-} H_{-} S H_{-}^{-1} A_{-}^{-1} M_{\left(z_{0, z_{3}}\right)} \text {. }
$$

Using the result in Lemma 1 and the fact that ${ }^{1} A_{-}$is in $W L_{p}^{n}\left(\gamma_{1}\right)$ we conclude that this last operator is bounded on $L_{p}^{n}\left(\gamma_{0}\right)$. It follows that ${ }^{0} A_{-}$is in $W L_{p}^{n}\left(\gamma_{0}\right)$ and this completes the proof.

4. PROOF OF THE THEOREM. Using a covering argument and the result in Lemma 2 we arrive at a local factorization of $A$ relative to $L_{p}$ on the whole contour $C$. This means that there are annular regions $\Omega_{0}^{ \pm} \subset D^{ \pm}$whose boundaries consist of $C$ and Liapounov contours $C^{ \pm}$and a factorization $A={ }^{0} A_{-}{ }^{0} A_{+}$on $C$ where the functions ${ }^{0} A_{ \pm}$and ${ }^{0} A_{ \pm}^{-1}$ are analytic on $\Omega_{0}^{ \pm}$. Moreover, the domains $\Omega_{0}^{ \pm}$can be cut into simply connected domains $\Omega_{1}^{ \pm}$ and $\Omega_{2}^{ \pm}$by a pair of Liapounov curves perpendicular to $C$ and $C^{ \pm}$such that ${ }^{0} A_{+} \in E_{q}^{n, n}\left(\Omega_{i}^{+}\right),{ }^{0} A_{-} \in E_{p}^{n, n}\left(\Omega_{i}^{-}\right),{ }^{0} A_{+}^{-1} \in E_{p}^{n, n}\left(\Omega_{i}^{+}\right)$and ${ }^{0} A_{-}^{-1} \in E_{q}^{n, n}\left(\Omega_{i}^{-}\right)$ $(i=1,2)$. Further the function ${ }^{0} A_{-}$is in $W L_{p}^{n}(C)$.

Choose a Liapounov contour $C^{\prime}$ between $C^{+}$and $C$ in $\Omega_{0}^{+}$which surrounds $C^{+}$. We can assume $0 \notin \Omega_{0}^{+}$. The function ${ }^{0} A_{+}$is invertible and smooth on $C^{\prime}$. Let ${ }^{0} A_{+}=G_{-} U_{1} G_{+}$be a factorization of ${ }^{0} A_{+}$relative to $C^{\prime}$.

The function

$$
\hat{A}_{+}(z)= \begin{cases}U_{1}^{-1} G_{-}^{-1}{ }^{0} A_{+}(z), & z \in\left[\operatorname{ext}\left(C^{\prime}\right) \cup C^{\prime}\right] \cap D^{+}, \\ G_{+}(z), & z \in \operatorname{int}\left(C^{\prime}\right),\end{cases}
$$

is analytic on $D^{+}$. Further, $\hat{A}_{+}^{-1}$ is analytic in $D^{+}, \hat{A}_{+} \in L_{q}^{n, n}(C)$ and $\hat{A}_{+}^{-1} \in L_{p}^{n, n}(C)$. Using property $3^{\circ}$ it is easy to conclude that $\hat{A}_{+} \in$ $\left[L_{q}^{n, n}(C)\right]^{+}$and $A_{+}^{-1} \in\left[L_{p}^{n, n}(C)\right]^{-}$. Define $\hat{A}_{-}={ }^{0} A_{-} G_{-} U_{1}$. The function $\hat{A}_{-}^{q}$ has all the properties of ${ }^{0} A_{-}$.

Next choose a Liapounov contour $C^{\prime \prime}$ between $C$ and $C_{-}$that surrounds $C$. Let $\hat{A}_{-}=F_{-} U F_{+}$be a factorization of $\hat{A}_{-}$relative to the contour $C^{\prime \prime}$. Set $A_{-}=\hat{A}_{-} F_{+}^{-1} U^{-1}$ and $A_{+}=F_{+} \hat{A}_{+}$. The factorization $A=A_{-} U A_{+}$is a factorization relative to $L_{p}(C)$. This completes the proof.

We would like to mention that the general scheme of the proof of the above theorem is analogous to the method used for the factorization relative 
to the contour of continuous functions (see [10, p. 102, p. 406]).

3. Factorization and other problems. An operator $T$ on a Banach space $X$ is said to be a Fredholm operator in case the range of $T$ is closed and the dimensions of the kernels of $T$ and $T^{*}$ are finite. It is well known that the operator $T$ on $X$ is Fredholm if and only if there are bounded operators $R$ and $L$ such that $T R=I+K$ and $L T=I+K^{\prime}$, where $K$ and $K^{\prime}$ are compact operators.

For the case of singular integral operators on $L_{p}^{n}(C)(1<p<\infty)$ there is the following description of Fredholm behavior. Let $A$ be in $L_{\infty}^{n, n}(C)$ and $p$ be fixed $(1<p<\infty)$. The singular integral operator $T_{A}=A P+Q$ is Fredholm on $L_{p}^{n}(C)$ if and only if the matrix function $A$ is invertible in $L_{\infty}^{n, n}(C)$ and admits a factorization relative to $L_{p}(C)$ [15], [17].

There exist local principles in the theory of Fredholm operators (see e.g. [3], [4], [10] and [14]). An operator $T$ in $L_{p}^{n}(C)(1<p<\infty)$ is called locally Fredhoim at the point $t_{0} \in C$ in case there is a neighborhood $\gamma_{0}$ of $t_{0}$ on $C$ and operators $R_{0}, L_{0}$ such that $M_{\gamma_{0}} T R_{0}=M_{\gamma_{0}}+K$ and $L_{0} T M_{\gamma_{0}}=M_{\gamma_{0}}+K^{\prime}$, where $K, K^{\prime}$ are compact operators and $M_{\gamma_{0}}$ denotes the operator of multiplication by the characteristic function of $\gamma_{0}$. It is known that the singular integral operator $T_{A}$ on $L_{p}^{n}(C)(1<p<\infty)$ is a Fredholm operator if and only if the operator $T_{A}$ is locally Fredholm at each $t_{0}$ in $C$ [14]. Another approach to this last result and an independent proof (when $p=2$ ) is in [5].

It is natural to compare for the singular integral operator $T_{A}=A P+Q$ on $L_{p}^{n}(C)(1<p<\infty)$ the notion that $T_{A}$ is locally Fredholm at a fixed point $t_{0} \in C$ with the notion that $A$ admits a local factorization at $t_{0}$ relative to $L_{p}$. The following is an unsolved problem.

Problem 1. Let $A$ be an invertible element in $L_{\infty}^{n_{1} n}(C), p$ fixed $(1<p<$ $\infty)$ and $t_{0}$ a fixed point in $C$. Does $A$ admit a local factorization relative to $L_{p}$ at $t_{0}$ if and only if the operator $T_{A}=A P+Q$ on $L_{p}^{n}(C)$ is locally Fredholm at $t_{0}$ ?

As comments on the above problem we make the following remarks. If $A$ is a function in $L_{\infty}^{n, n}(C)$ that admits a local factorization relative to $L_{p}$ on an arc $\gamma_{0}$ containing $t_{0}$ and if in $\gamma_{0}$ there are points $t_{1}, t_{2}$ on different sides of $t_{0}$ where the function $A$ is continuous, then the operator $T_{A}$ on $L_{p}^{n}(C)$ is locally Fredholm at $t_{0}$. Conversely, if the operator $T_{A}$ on $L_{p}^{n}(C)$ is locally Fredholm in a neighborhood of $t_{0}$ and there are points $t_{1}$ and $t_{2}$ on both sides of $t_{0}$ in this neighborhood where $A$ is continuous, then $A$ admits a local factorization at $t_{0}$ relative to $L_{p}$. The first remark is proved as follows. We can replace $A$ by any function that agrees with $A$ in a neighborhood of $t_{0}$. In this way we can assume $A$ is continuous everywhere on $C$ except on the open interval $\gamma^{\prime}$ between $t_{1}$ and $t_{2}$ containing $t_{0}$. It is well known that such an $A$ admits a local factorization relative to $L_{p}$ at every point not in $\gamma$. It follows from the main 
theorem that $A$ admits a factorization relative to $L_{p}(C)$ and, therefore, $T_{A}$ is locally Fredholm at $t_{0}$. The proof of the second remark made above is similar. Actually it is not necessary to assume that $A$ is continuous at $t_{1}$ and $t_{2}$ in the above remarks. The assumption that $A$ is continuous at $t_{1}$ and $t_{2}$ could be replaced by the assumption that $A$ is "locally sectorial" at $t_{1}$ and $t_{2}$ (see e.g. [14]).

The above argument leads to the following definition. Let $A$ be an invertible element in $L_{\infty}^{n, n}(C), p$ fixed $(1<p<\infty)$ and $t_{0}$ a fixed point in $C$. The matrix function $A$ is said to admit a restricted factorization relative to $L_{p}(C)$ at $t_{0}$ in case one can extend the restriction of $A$ in a neighborhood of $t_{0}$ to an element $A_{0}$ in $L_{\infty}^{n, n}(C)$ where $A_{0}$ admits a factorization relative to $L_{p}(C)$. The proof of the first remark made above shows that if $A$ admits a local factorization relative to $L_{p}$ at $t_{0}$ and there are points $t_{1}, t_{2}$ in $\gamma_{0}$ on both sides of $t_{0}$ such that $A$ is continuous at $t_{1}, t_{2}$, then $A$ admits a restricted factorization relative to $L_{p}(C)$ at $t_{0}$. More generally we ask:

Problem 2. Let $A$ be an invertible element in $L_{\infty}^{n, n}(C), p$ fixed $(1<p<$ $\infty$ ) and $t_{0}$ a fixed point in $C$. If $A$ admits a local factorization relative to $L_{p}$ at $t_{0}$, then does $A$ admit a restricted factorization relative to $L_{p}(C)$ at $t_{0}$ ?

Similarly, we make the following definition. Let $T_{A}=A P+Q$ be the singular integral operator on $L_{p}^{n}(C)(1<p<\infty)$ and $t_{0} \in C$. The operator $T_{A}$ will be called a restricted Fredholm operator at $t_{0}$ in case there is an $A_{0} \in L_{\infty}^{n, n}(C)$ such that $A=A_{0}$ in a neighborhood of $t_{0}$ and $T_{A_{0}}$ is a Fredholm operator on $L_{p}^{n}(C)$.

Obviously, $A$ admits a restricted factorization relative to $L_{p}$ at $t_{0}$ if and only if $T_{A}$ on $L_{p}^{n}(C)$ is a restricted Fredholm operator at $t_{0}$.

We raise one final question.

Problem 3. Let $T_{A}=A P+Q$ be a singular integral operator on $L_{p}^{n}(C)$ $(1<p<\infty)$ where $A \in L_{\infty}^{n, n}(C)$ and $t_{0}$ a fixed point in $C$. If the operator $T_{A}$ is locally Fredholm at $t_{0}$, then is $T_{A}$ a restricted Fredholm operator at $t_{0}$ ?

In conclusion we mention that the above three problems are unsolved when $n=1$ and $p=2$. Further the main theorem of the paper is true when one works on $L_{p}$ spaces with weights (see e.g. [10]) and the domain $D^{+}$is multiply connected.

ADDED IN PROOF. The authors have given affirmative answers to the three problems stated in $\S 3$ in the case where $n=1$ and $p=2$. This partial solution to Problem 1 depends on knowledge of the qualitative behavior of the factors in factorizations of continuous functions. No analogue of this behavior seems to be known in the matrix situation.

\section{REFERENCES}

1. M. S. Budjanu and I. C. Gohberg, General theorems on the factorization of matrix-valued functions. I. The fundamental theorem, Mat. Issled. 3 (1968), no. 2, 87-103; English transl.; Amer. 
Math. Soc. Transl. (2) 102 (1973), 1-14. MR 41 \# 4246a; 48 \#6.

2. General theorems on the factorization of matrix-valued functions. II. Some tests and their consequences, Mat. Issled. 3 (1968), no. 3, 3-18; English transl., Amer. Math. Soc. Transl. (2) 102 (1973), 15-26. MR 41 \#4246b; 48 \#6.

3. R. G. Douglas, Banach algebra techniques in operator theory, Academic Press, New York, 1972.

4. __ Local Toeplitz operators (preprint).

5. R. G. Douglas and D. E. Sarason, Fredholm Toeplitz operators, Proc. Amer. Math. Soc. 26 (1970), 117-120. MR 41 \# 4275.

6. P. L. Duren, The theory of $H^{p}$ spaces, Academic Press, New York, 1970. MR 42 \#3552.

7. I. C. Gohberg, The factorization problem in normed rings, functions of isometric and symmetric operators and singular integral equations, Uspehi Mat. Nauk 19 (1964), no. 1 (115), 71-124 = Russian Math. Surveys 19 (1964), no. 1, 63-114. MR 29 \#487.

8. I. C. Gohberg and I. A. Feldman, Projections methods for solving Weiner-Hopf equations, English transl; Math. monographs, vol. 41, Amer. Math. Soc., Providence, R. I., 1974. MR 50 \#8149.

9. I. C. Gohberg and M. G. Krein, Systems of integral equations on a half line with kernels depending on the difference of arguments, Uspehi Mat. Nauk 13 (1958), no. 2 (80), 3-72; English transl., Amer. Math. Soc. Transl. (2) 14 (1960), 217-287. MR 21 \# 1506; MR 22 \#3954.

10. I. C. Gohberg and N. Ya Krupnik, Introudction to the theory of one-dimensional singular integral operators, "Stiinca", Kishinev, 1973. (Russian)

11. G. M. Goluzin, Geometric theory of functions of a complex variable, English transl; Transl. Math. Monographs, vol. 26, Amer. Math. Soc., Providence, R. I., 1969. MR 40 \#308.

12. N. I. Mushelišvili, Singular integral equations. Boundary problems of function theory and their application to mathematical physics, 2nd ed., Fizmatgiz, Moscow, 1962; English transl. of 1st ed., Noordhoff, Groningen, 1953; reprinted, 1972. MR 15, 434; 50 \# 7968.

13. M. A. Shubin, On the local principle in the problem of factorization, Mat. Issled 6 (1971), no. $1,174-180$.

14. I. B. Simonenko, A new general method of investigating linear operator equations of the type of singular integral equations. I, Izv. Akad. Nauk SSSR Ser. Mat. 29 (1965), 567-586. (Russian) MR 31 \#3876.

15. , Some general questions in the theory of Riemann boundary problems, Izv. Akad. Nauk SSSR 32 (1968) = Math USSR Izv. 2 (1968), 1091-1099.

16. H. Röhrl, On holomorphic families of fiber bundles over the Riemann Sphere, Mem. Coll. Sci. Univ. Kyoto Ser. A Math. 33 (1960/61), 435-477. MR 24 \# A1728.

17. H. Widom, Singular integral equations in $L_{p}$, Trans. Amer. Math. Soc. 97 (1960), 131-160. MR 22 \#9830.

Department of Mathematics, University of Georgia, Athens, Georgia 30602

Department of Mathematics, University of Tel Aviv, Tel Aviv, Israel

Department of Mathematics, State University of New York at Stony Brook, Stony BROOK, NEW YORK 11794 